\title{
sciendo
}

\section{Relationship Between Toe Muscular Strength and the Ability to Change Direction in Athletes}

\author{
by \\ Yasuhiro Yuasa ${ }^{1}$, Toshiyuki Kurihara ${ }^{1}$, Tadao Isaka ${ }^{1}$
}

This study aimed to investigate the relationship between toe muscular strength and the ability to change direction in athletes. Seventeen collegiate American-football players participated in the study (age $19.9 \pm 0.9$ years, competition experience $7.3 \pm 1.7$ years). Two types of measurements were performed to evaluate toe muscular strength: toe flexor strength with the metatarsophalangeal joint in the planter flexed position and toe-pushing force with the metatarsophalangeal joint in the dorsiflexed position. The ability to change direction was evaluated using the pro-agility and 3-cone tests and change of direction deficits, calculated by subtracting the sprint times from the pro-agility and 3cone times. There were significant correlations between toe-pushing force and the results of the pro-agility and 3-cone tests, but no significant correlations between toe flexor strength and the pro-agility and 3-cone tests. Neither toepushing force nor toe flexor strength was significantly correlated with the sprint test results. Furthermore, toe-pushing force was significantly correlated with the 3-cone test deficit, but toe flexor strength was not. The ability to change direction is more strongly affected by toe muscular strength (measured as toe-pushing force) with the metatarsophalangeal joint in the dorsiflexed angle than by toe muscular strength (measured as toe flexor strength) with the metatarsophalangeal joint in the plantar flexed angle. Our results suggest that athletes can improve their ability to change direction with toe muscular strength training with the metatarsophalangeal joint in the dorsiflexed position.

Key words: metatarsophalangeal joint, agility, physical tests, athletic performance.

\section{Introduction}

Changing the direction of movement is a frequent sight in various sports and is believed to be a key factor in athletic performance. In a previous study, more than 700 different situations in which a change in direction was necessary were observed in a single soccer game (Bloomfield et al., 2007). These findings suggest that in field sports, the ability to change direction quickly is as important as the ability to sprint fast or jump high.

It is well known that there is a positive correlation between the speed at which a person can change the direction and their straight sprinting speed (Gabbett et al., 2008; Little and Williams, 2005; Sassi et al., 2009). Some previous studies focusing on the ability to change the direction have focused mainly on the effect of the extensor muscles of the lower limb joints, such as those of the hips, knees, and ankles (Mikołajec et al., 2017; Markovic, 2007; Newman et al., 2004; Young et al., 2002). However, these previous studies neglected the most distal joints of the lower limb, such as the metatarsophalangeal joints (MPJs), which are located at the forefoot. Although the moment produced by the MPJ is smaller than that of the other lower-limb joints, the forefoot is the only part of the body touching the ground during changes in direction. Moreover, the dorsiflexion moment of the MPJ has been observed during the late-stance phase of changes in direction (Riley et al., 2013). Therefore, the moment of the MPJ may play an important role in conveying the force of the larger extensor muscles to the ground during changes in

1 - Department of Sport and Health Science, Ritsumeikan University, Japan. 
direction.

The MPJ undergoes dorsiflexion at the mid-stance to the push-off phase during sprinting (Smith et al., 2014). When the forefoot touches the ground during changes in direction, the MPJ is in a dorsiflexed position rather than a plantar flexed position. Previous studies have demonstrated that toe muscular strength (TMS) depends on the angles of the MPJ and the ankle joint, which determine the force-length relationship of the extrinsic foot muscles (Goldmann and Brüggemann, 2012; Goldmann et al., 2013). Most previous studies have measured TMS with the MPJ in the plantar flexed position (Morita et al., 2015; Otsuka et al., 2015), while TMS is at its maximum when the MPJ is in the dorsiflexed position (Goldmann and Brüggemann, 2012). Thus, to verify the contribution of TMS to athletic performance, it should be measured when the MPJ is dorsiflexed. No studies have investigated these relationships to date. With this in mind, the aim of this study was to investigate the relationship between TMS and the ability to change direction in athletes. We hypothesized that TMS with the MPJ in the dorsiflexed position would indicate the ability to change direction better than TMS with the MPJ in the plantar flexed position.

\section{Methods}

\section{Participants}

Seventeen collegiate American-football players (age $19.9 \pm 0.9$ years, competition experience $7.3 \pm 1.7$ years) participated in the present study. All participants were members of the winning team of the 2015 Japanese National College Football Championship and played in wide receiver (WR) or running back (RB) positions in the season just before the experiment was performed. The methods and all procedures were in accordance with current local guidelines and approved by the Research Ethics Committee Involving Living Human Participants at the Ritsumeikan University (IRB-BKC-2015-002). All participants were informed about the procedures, risks, and purposes of the study, and provided consent before participation.

\section{Measures}

Toe muscular strength (TMS)

Two types of apparatus were used for the evaluation of TMS. First, toe flexor strength (TFS) was measured according to previous studies (Morita et al., 2015; Otsuka et al., 2015) using a commercially available dynamometer (T.K.K. 3361, Takei Scientific Instrument Co., Niigata, Japan). Second, toe-pushing force (TPF) was measured using a custom-made dynamometer (Rowley et al., 2015) (T.K.K. 1268, Takei Scientific Instrument Co., Niigata, Japan). Both TFS and TPF were measured three times for both the right and left feet in randomized order. The biggest value from the three trials was normalized by body mass and used for further analysis.

Measurement of the ability to change direction, acceleration, and sprint speed

The ability to change direction was evaluated using pro-agility and 3-cone tests according to previous studies (McGee and Burkett, 2003; Robbins, 2010). Acceleration and speed sprint were evaluated using the 10- and 40-yard sprint tests. From these measurements, we calculated change of direction deficits.

The 10- and 40-yard sprint tests were performed using electronic timing devices (Brower Timing System, Salt Lake City, Utah). All participants attempted to sprint to the 10 and 40 yard lines twice, and their times were recorded to the accuracy of $0.01 \mathrm{~s}$. The best time of the two attempts was entered into the final analysis.

The change of direction deficit was calculated by subtracting the sprint time from the results of the change of direction tests (Nimphius et al., 2013). A previous study demonstrated a significant correlation between 10-yard sprint time and 3-cone test time (Gabbett et al., 2008). Thus, the change of direction deficit of the 3-cone test was calculated as the difference between the times of the 3-cone test and the 10-yard sprint test. The change of direction deficit of the pro-agility test was calculated by subtracting the 10-yard sprint time from the halved time recorded on the pro-agility test.

\section{Design and Procedures}

Toe muscular strength (TMS)

Toe flexor strength was measured with the MPJ in the plantar flexed position. In short, the participants sat with their hip, knee, and ankle joints at $90^{\circ}$. The participants were then instructed to put their toes on a grip bar and pull it with maximum effort (Figure 1A). Toe-pushing force was measured with the MPJ in the dorsiflexed position. The participants were seated on a chair 
with a posture similar to that used for the measurement of TFS (Figure 1B). The hip, knee, and ankle joints were set at $90^{\circ}$ during maximal voluntary isometric contractions (MVICs). The participants were then instructed to place their toes on an adjustable force plate with the MPJ at a $45^{\circ}$ dorsiflexed angle. They were asked to use only the toe muscles and not to activate the calf muscles. A 1-min rest was allowed between each measurement.

Measurement of the ability to change direction, acceleration, and sprint speed

All measurements were performed on a football field on a different day to TMS measurements. As for a warm-up, it has been suggested that a short-term static stretching session has a positive influence on agility performance (Amiri-Khorasani et al., 2010), thus a warm-up was carried out in an identical manner for all subjects with 5 minutes of static stretching to the whole body and 5 minutes of dynamic activity.

The pro-agility test involved performing acceleration and deceleration during quick turning movements (Figure 2A). The participant began in a 3-point stance with the legs across the start line and one hand on the start line; this pose was held for at least $1 \mathrm{~s}$. After a signal was given, the participant immediately ran to the right (or left) and touched the right (or left) 5-yard line with the right (or left) hand, then quickly changed direction to the left (or right) and touched the left (or right) 5-yard line with the left (or right) hand, finally running through the finish line. Each participant was allowed two attempts. The assessor chose the direction in which the participant ran first.

The 3-cone test was similar to the pro-agility test (Figure 2B). Three cones were set at 5-yard intervals to form an "L" shape. The participants started the test in a 3-point stance behind the starting line and put one hand on the line. Once a signal was given, the participant sprinted 5 yards forward, touched the line and returned to the start line. Then, the participant sprinted toward cone 2 and turned to sprint again toward cone 3 . The participant circled cone 3 and returned to the finish line. The participant was not allowed to touch the cones or place their hands on the floor when making turns or circling the final cone. All participants executed two attempts with cone 3 on the left and two attempts with the cone on the right side.

\section{Statistical Analysis}

All data were presented in the form of means \pm standard deviations. Comparisons between the right and left sides were examined by paired $\mathrm{t}$ tests. The intra-rater reliability of TFS, TPF, and athletic performance measurements were evaluated using intra-class correlation coefficients (ICCs). Relationships between all test variables (TFS, TPF, pro-agility test, 3-cone test, 10-yard sprint test, 40-yard sprint test, and the change of direction deficits of the pro-agility and 3-cone tests) were examined by the Pearson's correlation coefficient. The level of statistical significance was set at $\mathrm{p}<0.05$.

\section{Results}

The physical characteristics and the results of TMS and performance tests are summarized in Table 1 . The ICCs $(1,3)$ of TFS and TPF on the left and right sides ranged from 0.73 to 0.81 . The difference between left and right sides was not significant for either TFS or TPF (TFS: $p=0.10, \mathrm{~d}=$ 0.28 ; TPF: $p=0.26, \mathrm{~d}=0.22)$. The ICCs $(1,2)$ of the pro-agility, 3-cone, 10-yard sprint, and 40-yard sprint tests ranged from 0.61 to 0.81 , and the proagility and 3-cone tests were not significantly different for the left and right sides (pro-agility test: $p=0.27, \mathrm{~d}=0.16$; 3-cone test: $p=0.22, \mathrm{~d}=$ $0.20)$. Then, the values of both sides were averaged. Figure 3 shows the correlations among TFS/TPF and the pro-agility and 3-cone tests. Toepushing force was negatively correlated with proagility and 3-cone test results $(r=-0.503$ and -0.498 , respectively, $p<0.05$ ), but TFS was not significantly correlated with pro-agility or 3-cone test results $(\mathrm{r}=-0.398, p=0.11$ and $\mathrm{r}=-0.410, p=$ 0.10 , respectively). There were no significant correlations between TPF/TFS and 10-yard or 40yard sprint test results (TPF with 10- and 40-yard sprint: $\mathrm{r}=-0.317, p=0.22$ and $\mathrm{r}=-0.356, p=0.16$, respectively; TFS with 10- and 40-yard sprint: $\mathrm{r}=$ $-0.288, p=0.26$ and $\mathrm{r}=-0.343, p=0.18$, respectively). Finally, TFS was not significantly correlated with pro-agility or 3-cone test deficits ( $\mathrm{r}$ $=-0.139, p=0.60$ and $\mathrm{r}=-0.390, p=0.12$, respectively). However, TPF was negatively correlated with the 3-cone deficit $(\mathrm{r}=-0.482, p<$ $0.05)$, but not significantly correlated with the proagility deficit $(\mathrm{r}=-0.210, p=0.42)$. 
(A)

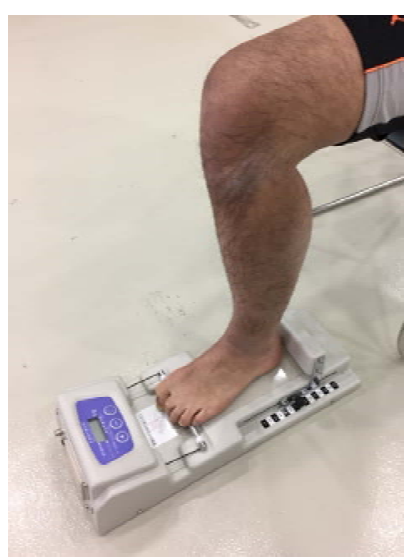

(B)

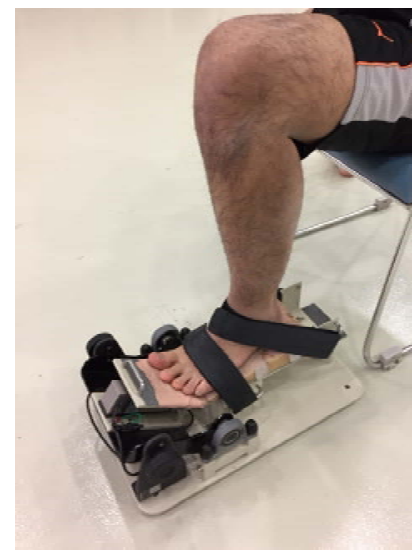

Figure 1

Measurement of toe muscular strength.

Toe flexor strength is measured with the ankle joint in the neutral position and the metatarsophalangeal joints (MPJs) in the plantar flexed position (A).

Toe-pushing force is measured with the ankle joint in the neutral position and the MPJ in the $45^{\circ}$ dorsiflexion position (B)

\section{(A)}

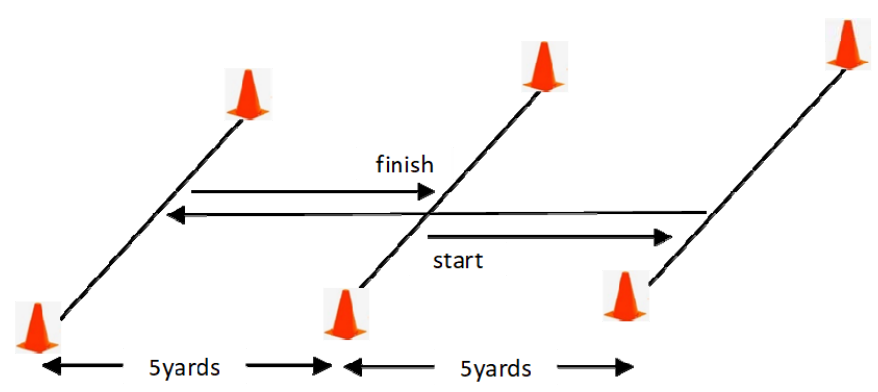

(B)

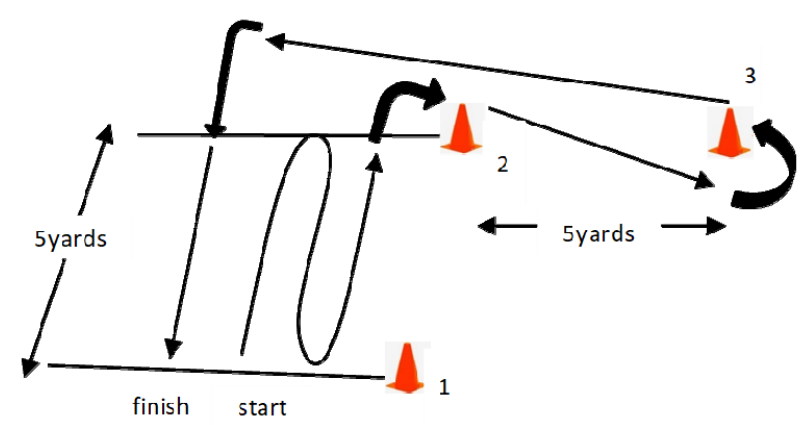

Figure 2

Schematic diagram of the measurement of ability to change direction

Pro-agility test (A) and 3-cone test (B). 

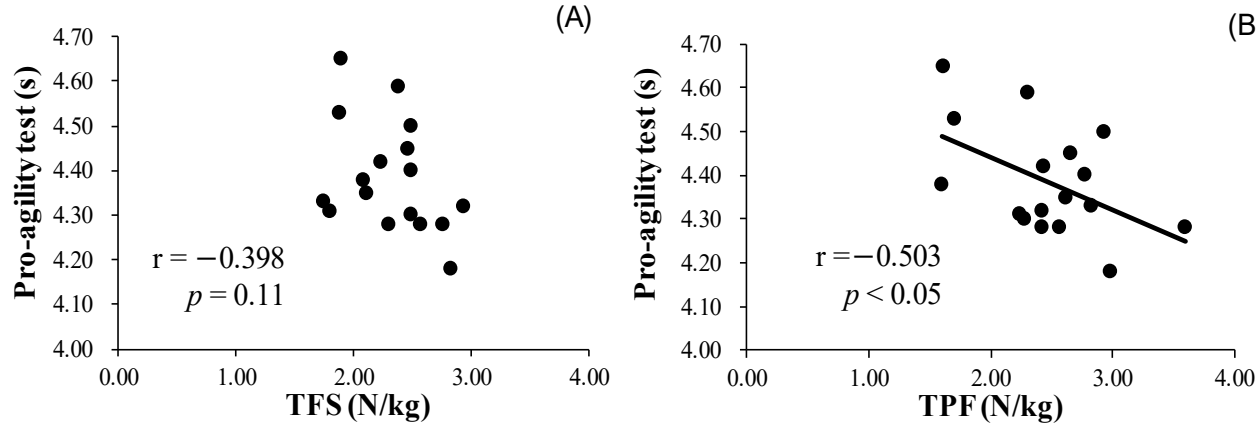

(B)
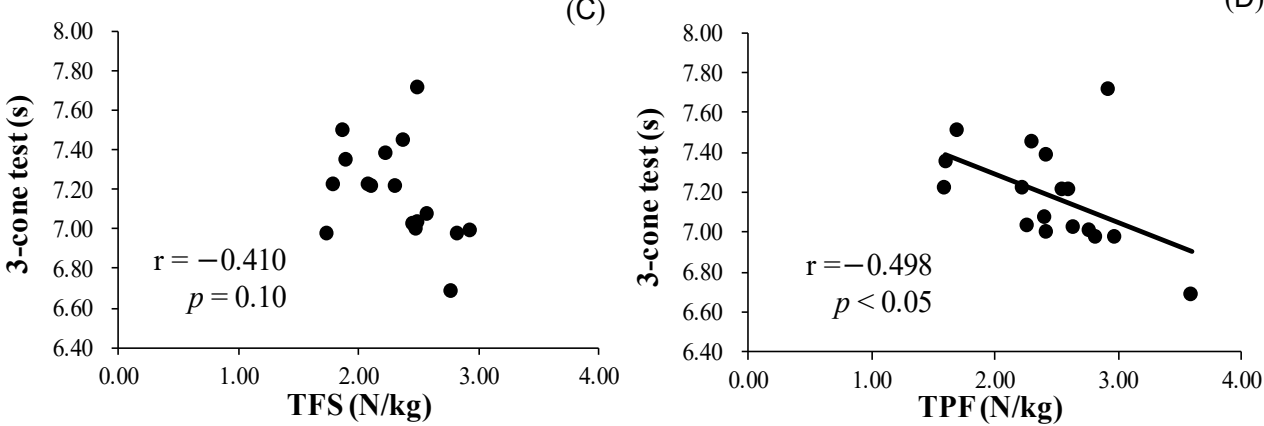

Figure 3

Correlations between toe muscular strength and the ability to change direction The figure shows correlations between toe flexor strength (TFS) / toe-pushing force (TPF) and pro-agility test results $(A, B)$, and 3-cone test results $(C, D)$.

Table 1

Physical characteristics, TFS, TPF, pro-agility test, 3-cone tests, 10-yd dash, and 40-yd dash tests

\begin{tabular}{lccc}
\hline & & mean $\pm \mathrm{SD}$ & ICC \\
\hline Body Height $(\mathrm{cm})$ & & $173.0 \pm 7.0$ & \\
Body mass $(\mathrm{kg})$ & left & $2.18 \pm 0.44$ & left 0.73 \\
Toe flexor strength (N/kg) & right & $2.28 \pm 0.37$ & right 0.75 \\
& left & $2.24 \pm 0.57$ & left 0.81 \\
Toe pushing force (N/kg) & right & $2.35 \pm 0.44$ & right 0.76 \\
& left & $4.43 \pm 0.13$ & 0.76 \\
Pro agility test (sec) & right & $4.41 \pm 0.12$ & \\
& left & $0.50 \pm 0.07$ & 0.79 \\
Pro agility deficit (sec) & right & $0.49 \pm 0.06$ & 0.81 \\
& left & $7.22 \pm 0.28$ & \\
3 cone test (sec) & right & $7.27 \pm 0.21$ & 0.77 \\
& left & $5.51 \pm 0.25$ & 0.61 \\
3 cone deficit (sec) & right & $5.56 \pm 0.19$ & 0.77 \\
10-yd dash (sec) & & $1.71 \pm 0.06$ & \\
40 -yd dash (sec) & & $5.01 \pm 0.14$ & \\
\hline
\end{tabular}

All data are expressed as means \pm standard deviation (S.D.).

* : significant differences between left and right $(p<0.05)$ 


\section{Discussion}

This was the first study to investigate the relationship between TMS and the ability to change direction in athletes. The major finding was a significant correlation between the ability to change direction and maximum TMS with the MPJ in the dorsiflexed position (TPF), but no significant correlation between the ability to change direction and maximum TMS with the MPJ in the plantar flexed position (TFS). Moreover, the change in direction deficits indicated that TPF was more closely correlated with the ability to change direction than TFS according to the 3-cone deficit. Our results suggest that the measurement of TMS with the MPJ in the dorsiflexed position (using TPF) is meaningful for the evaluation of the ability to change direction in athletes.

As described above, the ability to change direction was significantly correlated with TPF, but not with TFS. These findings support our hypothesis that TPF is a better indicator of the ability to change direction than TFS. The dorsiflexion movement of the MPJ causes the plantar fascia to wind around the metatarsal heads, which is known as the "windlass mechanism" (Kappel-Bargas et al., 1998). Toe muscular strength induces the maximal force because the truss structure of the foot increases the tension of the plantar fascia to produce greater force (Erdemir at al., 2004). This activates the muscles in the foot to produce force during the push-off phase of walking in humans (Hicks, 1954). However, the degree of correlation was slightly weak (TPF with pro-agility test: $\mathrm{r}^{2}=0.25$ and TPF with 3-cone test: $r^{2}=0.25$, respectively), and this was most likely because factors other than TMS play a role in the ability to change direction. The ability to change direction is mainly produced by the integrated muscle forces of the lower limb (Young et al., 2002). In fact, TMS is much lower (mean 2.2-2.4 N/kg in this study) than hip joint extension torque $(28.1 \mathrm{Nm} / \mathrm{kg})$, knee extension torque $(28.5 \mathrm{Nm} / \mathrm{kg})$ and ankle plantar flexion torque $(16.6 \mathrm{Nm} / \mathrm{kg}$ ) (Anderson et al., 2007). However, some previous studies have reported that the ability to change direction was negatively correlated with lower limb power and reactive strength of the leg (Nimphius et al., 2010; Young et al., 2002). In the stance phase of walking and running, muscles of the thigh, calf, and foot are activated in sequence (Cappellini et al., 2006), and toe flexor muscles are then activated in the late stance phase (Mann and Inman, 1964; Riley et al., 2013). The forefoot, the only body part touching the ground during changes in direction, may mechanically react to the joint moment and power of the lower limbs via a kinetic chain, working as the terminal point of the kinetic chain during human movement (Riley et al., 2013). This idea is supported by the fact that strength training for toe flexor muscles improves jumping performance (Goldmann et al., 2013; Unger and Wooden, 2000). These previous studies and the results of the present study suggest that TMS has a significant effect on athletic performance.

The agility tests performed in this study are frequently used in studies of athletic performance (McGee and Burkett, 2003; Robbins, 2010). Briefly, the pro-agility test, a simple test consisting of acceleration and deceleration phases during sprints (McGee and Burkett, 2003; Robbins, 2010), and the 3-cone test, a test including speed, agility, change of direction, body control, and power (McGee and Burkett, 2003; Robbins, 2010), are designed to assess turning techniques, leg muscle qualities, and straight sprinting speed (Young et al., 2002). These tests are often used for measuring agility of athletes. However, more simple tests for evaluating acceleration and speed sprint ability, the 10- and 40-yard sprint tests, were not significantly correlated with TMS in this study. The sprint test is similar to the pro-agility and 3-cone tests in terms of total distance ( 20 and 30 yards, respectively), but different in terms of the number of changes of direction. These results suggest that TMS could contribute to the ability to change direction rather than straight sprint speed. The change of direction deficit is intended to evaluate the ability to change direction independently from linear sprint capacity (Nimphius et al., 2013). The pro-agility deficit was introduced in a previous study (Nimphius et al., 2013), but the 3-cone deficit has not been investigated. The methodological differences between the proagility and 3-cone tests are related to the number of changes in direction and the types of turns. The pro-agility test includes two $180^{\circ}$ turns, while the 3 -cone test contains five turns, including two $90^{\circ}$ turns, two $180^{\circ}$ turns, and one $360^{\circ}$ turn around a cone. It is thought that these variations influence 
the results.

American football players are required to change their direction of movement quickly when confronting various situations in games. As a result, most performance testing for American football players focuses on high-intensity activities, such as linear sprints, the speed of changes in direction, and agility (McGee and Burkett, 2003; Robbins, 2010). The WR and RB are particularly skillful positions that require the player to run across the field while attempting to elude opponents and catch the ball. Thus, the ability to change direction quickly is very important to these players. The subjects in the present study had an average of seven years of experience playing football and had played in the WR or RB positions during the most recent season. For this reason, these players likely possessed sufficient fundamental skills in changing direction.

Based on the results of this study, TMS contributes to athletic performance, especially in athletes who need to change their direction quickly. However, the conventional exercises for strengthening intrinsic foot muscles, such as the towel-curl and short-foot exercise, have been performed at the MPJ in the plantar flexed position (Lynn et al., 2012). Therefore, it is recommended that athletes perform these exercises with the TMS at the MPJ in the dorsiflexed position, such as the jump rope and/or the calf raise exercises. Also, in practical situations, the use of spiked shoes makes it easy to change the direction of movement without any involvement of the forefoot. This is supported by the fact that the mean range of motion of the MPJ during sprinting is $51.5 \pm 3.5^{\circ}$ while barefoot and decreases to $42.3 \pm 5.7^{\circ}$ while wearing spiked shoes (Smith et al., 2014). Empirically, some athletes in this study who did not have good ability to change direction before the training session showed significant improvement after training while barefoot. The results of this study suggest that athletes can change direction more quickly if the toes are used. From these findings, other factors not evaluated in this study (e.g., shoes type, surface texture, etc.) should be also considered. Furthermore, if coaches evaluate the athlete's toe function, it is thought that it should be done with the MPJ in a dorsiflexed position.

The major limitation of this study was that it was cross-sectional in nature. In the future, it will be necessary to investigate whether TMS training is related to the improvements in the ability to change direction. Furthermore, no control subjects and only 17 participants were included. This was a small sample size, but we only enrolled athletes who played at the WR and RB positions of American football. These individuals have a good baseline ability to change direction, so it is not clear whether the results of this study can be generalized to other athletes or members of the general population. Finally, previous studies have reported that warming up with short-term static stretching has a positive influence on repeated sprint (less than $10 \mathrm{~s}$ ) and agility performance (Amiri-Khorasani et al., 2010; Marinho et al., 2017), so it is necessary to consider the effect of warming-up in the future.

In conclusion, the ability to change the direction of movement is significantly correlated with TMS with the MPJ in the dorsiflexed position. This finding suggests that TMS with the MPJ in the dorsiflexed position is a good indicator of the ability to change direction. Our results suggest that athletes can improve their ability to change direction with toe muscular strength training with the metatarsophalangeal joint in the dorsiflexed position.

\section{Acknowledgements}

We thank all the participants in the study. This work was supported by JSPS KAKENHI, Grant-in-Aid for Scientific Research (c) (\# JP26350825).

\section{References}

Amiri-Khorasani M, Sahebozamani M, Tabrizi KG, Yusof AB. Acute effect of different stretching methods on Illinois agility test in soccer players. J Strength Cond Res, 2010; 24(10): 2698-2704

Anderson DE, Madigan ML, Nussbaum MA. Maximum voluntary joint torque as a function of joint angle and angular velocity: Model development and application to the lower limb. J Biomech, 2007; 40: 31053113 
Bloomfield J, Polman R, O' Donoghue P. Physical demands of different positions in FA Premier League soccer. J Sport Sci Med, 2007; 6: 63-70

Cappellini G, Ivanenko YP, Poppele RE, Lacquaniti F. Motor patterns in human walking and running. J Neurophysiol, 2006; 95: 3426-3437

Erdemir A, Hamel AJ, Fauth AR, Piazza SJ, Sharkey NA. Dynamic loading of the plantar aponeurosis in walking. J Bone Joint Surg Am, 2004; 86(3): 546-552

Gabbett TJ, Kelly JN, Sheppard JM. Speed, change of direction speed, and reactive agility of rugby league players. J Strength Cond Res, 2008; 22(1): 174-181

Goldmann JP, Brüggemann GP. The potential of human toe flexor muscles to produce force. J Anat, 2012; 221: 187-194

Goldmann JP, Sanno M, Willwacher S, Heinrich K, Brüggemann GP. The potential of toe flexor muscles to enhance performance. J Sport Sci, 2013; 31(4): 424-433

Hicks JH. The mechanics of the foot. II. The plantar aponeurosis and the arch. J Anat, 1954; 88(1): 25-30

Kappel-Bargas A, Woolf RD, Cornwall MW, McPoil TG. The windlass mechanism during normal walking and passive first metatarsalphalangeal joint extension. Clin Biomech, 1998; 13(3): 190-194

Little T, Williams AG. Specificity of acceleration, maximum speed, and agility in professional soccer players. J Strength Cond Res, 2005; 19(1): 76-78

Lynn SK, Padilla RA, Tsang KK. Differences in static- and dynamic-balance task performance after 4 weeks of intrinsic-foot-muscle training: the short-foot exercise versus the towel-curl exercise. J Sport Rehabil, 2012; 21(4): 327-333

Mann R, Inman VT. Phasic activity of intrinsic muscles of the foot. J Bone Joint Surg Am, 1964; 46(3): 469-481

Marinho DA, Gil MH, Marques MC, Barbosa TM, Neiva HP. Complementing warm-up with stretching routines: effects in sprint performance. Sports Medicine International Open, 2017; 1(3): 101-106

Markovic G. Poor relationship between strength and power qualities and agility performance. J Sport Med Phys Fit, 2007; 47: 276-283

McGee KJ, Burkett LN. The National Football League combine: A reliable predictor of draft status? J Strength Cond Res, 2003; 17(1): 6-11

Mikołajec, Kazimierz, Maszczyk, Adam, Chalimoniuk, Małgorzata, Langfort, Józef, Gołaś, Artur, Zajc, Adam. The influence of strength exercises of the lower limbs on postural stability: A possible role of the autonomic nervous system. Isokinet Exerc Sci. 2017; 25(2): 79-89

Morita N, Yamauchi J, Kurihara T, Fukuoka R, Otsuka M, Okuda T, Ishizawa N, Nakajima T, Nakamichi R, Matsuno S, Kamiie S, Shide N, Kambayashi I, Shinkaiya H. Toe flexor strength and foot arch height in children. Med Sci Sport Exer, 2015; 47(2): 350-356

Newman MA, Tarpenning KM, Marino FE. Relationships between isokinetic knee strength, single-sprint performance, and repeated-sprint ability in football players. J Strength Cond Res, 2004; 18(4): 867-872

Nimphius S, McGuigan MR, Newton RU. Relationship between strength, power, speed, and change of direction performance of female softball players. J Strength Cond Res, 2010; 24(4): 885-895

Nimphius S, Geib G, Spiteri T, Carlisle D. "Change of direction" deficit measurement in Division I American football players. Jounal of Australian Strength and Conditioning, 2013; 21(S2): 115-117

Otsuka M, Yamauchi J, Kurihara T, Morita N, Isaka T. Toe flexor strength and lower-limb physical performance in adolescent. Gazzetta Medica Italiana, 2015; 174(7-8): 307-313

Riley PO, Kent RW, Dierks TA, Lievers WB, Frimenko RE, Crandall JR. Foot kinematics and loading of professional athletes in American football-specific tasks. Gait Posture, 2013; 38: 563-569

Robbins DW. The National Football League (NFL) combine: Does normalized data better predict performance in the NFL draft? J Strength Cond Res, 2010; 24(11): 2888-2899

Rowley KM, Jarvis DN, Kurihara T, Chang YJ, Fietzer AL, Kulig K. Toe flexor strength, flexibility and 
function and flexor hallucis longus tendon morphology in dancers and non-dancers. Med Probl Perform Ar, 2015; 30(3): 152-156

Sassi RH, Dardouri W, Yahmed MH, Gmada N, Mahfoudhi ME, Gharbi Z. Relative and absolute reliability of a modified agility T-test and its relationship with vertical jump and straight sprint. J Strength Cond Res, 2009; 23(6): 1644-1651

Smith G, Lake M, Lees A. Metatarsophalangeal joint function during sprinting: A comparison of barefoot and sprint spike shod foot conditions. J Appl Biomech, 2014; 30: 206-212

Unger CL, Wooden MJ. Effect of foot intrinsic muscle strength training on jump performance. J Strength Cond Res, 2000; 14(4): 373-378

Young WB, James R, Montgomery I. Is muscle power related to running speed with change of direction? J Sport Med Phys Fit, 2002; 42: 282-288

\section{Corresponding author:}

\section{Toshiyuki Kurihara}

Department of Sport and Health Science, Ritsumeikan University, Kusatsu, Japan

1-1-1 Noji Higashi, Kusatsu, Shiga 525-8577, Japan.

Phone: +81-77-561-3760

Fax: +81-77-561-3761

E-mail: t-kuri-a@st.ritsumei.ac.jp 